\title{
Studien über den Codex Arabicus Monacensis Aumer 238.
}

\author{
Von Karl Römer.
}

Die folgenden Untersuchungen bilden einen Teil meiner Arbeiten über die berühmte spanisch-arabische Evangelienhandschrift Aumer 238, die sowohl in technisch-philologischer wie auch in textkritischer Hinsicht ein eingehendes Studium verlohnt. Einen Abschnitt der technisch-formalen Arbeit über diesen Münchner Kodex konnte ich vor wenigen Monaten als Dissertation erscheinen lassen. Die nachstehenden Blätter enthalten die direkte Fortsetzung dieser Schrift, auf die der Leser des Zusammenhangs halber ausdrücklich verwiesen sei $^{\mathrm{I}}$ ) und $\mathrm{z}$ war zunächst den Schluss des grammatischen Teiles der dort (auf S. VII) näher gekennzeichneten "Abteilung II". Indessen beabsichtige ich, womöglich den gesamten "Ersten Hauptteil a in dieser Zeitschirift zum Abdruck zu bringen, sowie vom zweiten Hauptteil, der das betreffende Manuskript nach theologisch-textkritischen Gesichtspunkten behandelt, wenigstens die allgemeinen Resultate, sofern sie zum Gesamturteil über die Abfassung des Kodex etc. beigezogen werden müssen.

I) K. Römer, Der Codex Arabicus Monacensis Aumer 238. Eine spanisch-arabische Evangelienhandschrift. Leipzig (Drugulin) 1905. 


\section{Zur Formenlehre.}

Die Partikeln.

\section{§ 1. Die Präpositionen.}

Der Einheitlichkeit halber empfiehlt es sich auch hier, die syntaktischen Fragen zum Teil schon mit den formalen zu behandeln.

a) $\bar{c}$ wird wie in der Schriftsprache zeitlich verwendet: vespere autem facto: $41^{2}, 19$ فلنَّا كان مع الهَسَاء $\mu$ 14, I7;

ebenso 15b, 19. M 16,2: مع الهـاه vespere facto; ibid.: مع البل

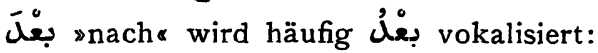

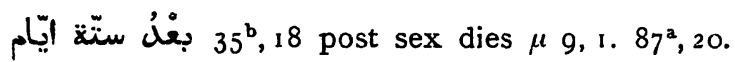
J 20, 26;

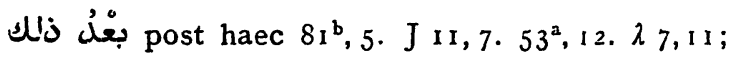

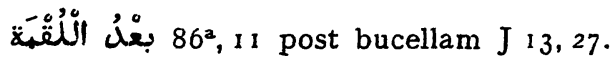

Vielleicht war dem Schreiber die Aussprache von بعل mit auslautendem Vokal nicht mehr geläufig, so dass hier eine Verwechslung mit dem adverbialen uُ vorliegt. Lerch. Vok. kennt bei der Aussprache keinen Endvokal mehr: después de heisst bei ihm de baâd.

Damma statt Fatha findet sich auch selten bei:

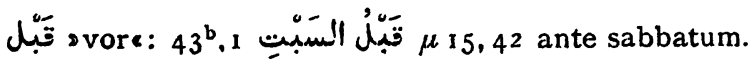


In folgenden Fällen kommen zwei Präpositionen mit einander verbunden vor:

تنتى إلى الساءة -

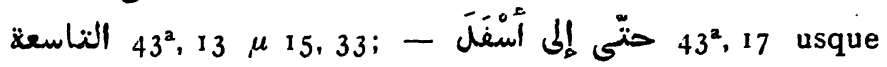
deorsum $\mu 15,38$.

Dasselbe gilt von dem sehr häufig präpositional gegrauchten

جُوار iuxta, welches bald ohne vorgesetzte Präposition in diesem Sinn mit dem Nomen verbunden wird, bald mit

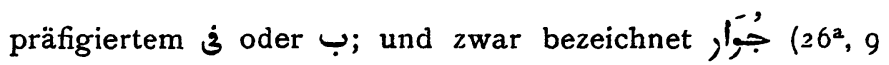
جَمَ $\mathrm{M} 27,46)$ die Nähe sowohl im Raum als in der Zeit. Der Vokabulista kennt diese Präposition in der Form $=$ iuxta. Beispiele in der Verbindung sind:

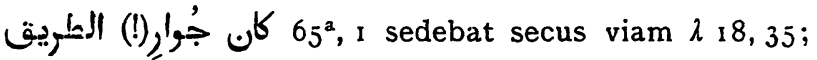
ebenso $\mathrm{IV}^{\mathrm{b}} 9 \mathrm{MXX} ; 3^{\mathrm{a}}, 7 \mu$ 10, 46;

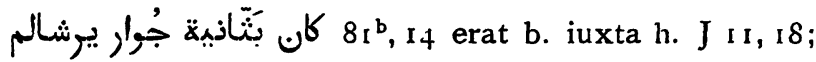

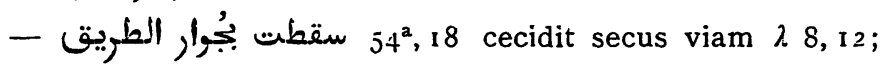
- 542a 12 it. 2.8,5;

ebenso $=\operatorname{circa} 31^{\mathrm{a}}, 18 \mu 4,15 ; 65^{\mathrm{b}}, 8 \lambda \quad 19,29 ;-$

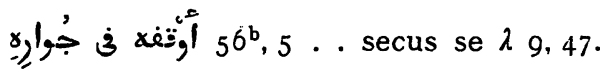

Der nominale Charakter von جُو zu Tage in den Verbindungen: partes tyri MI I5, 2 I; - 9 erat autem proximum pascha .. iudaeorum J 6, 4; $77^{\mathrm{b}}$, 10; $\mathrm{J} 7,2$.

b) Ueber den abweichenden Gebrauch einzelner Präpositionen wird an den betreffenden Stellen des Vokabu- 
Studien über den Codex Arabicus Monacensis Aumer 238. IO I lars gehandelt werden; hier sollen nur einzelne Beispiele angeführt werden:

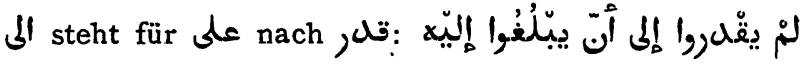
$54^{\mathrm{b}}, 8$ non poterent adire ad eum $\lambda 8,19$.

إلى (على ) werden gebraucht statt des Akkusativs bezw. bei أضَ إنَ scandalizare, detrimentum facere; (يلك)

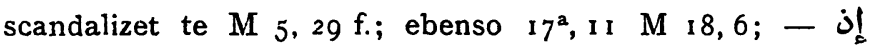

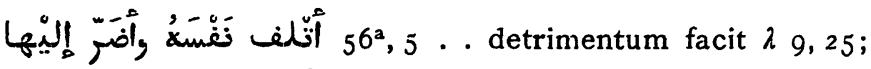

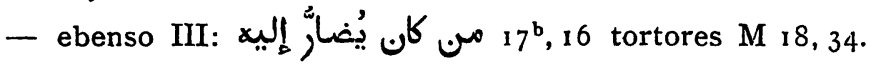

c) Verbindung der Präposition mit ihrem Nomen.

Das von der Präposition abhängige Nomen steht sehr häufig im Nominativ oder Akkusativ, in andern Fällen bleibt jede Kasusendung fort und wird durch Ġezma ersetzt. Beispiele sind:

a) إلى 20a , 20 (ite) ad exitus viarum M 22, 9; -

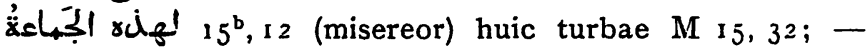

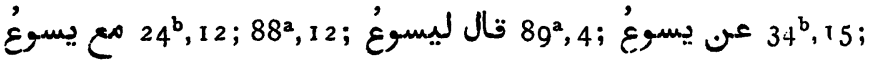

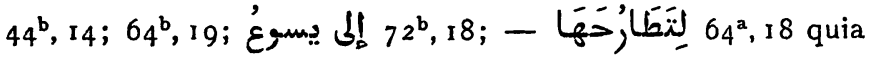

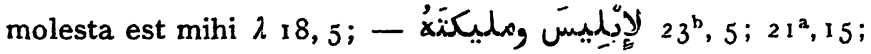

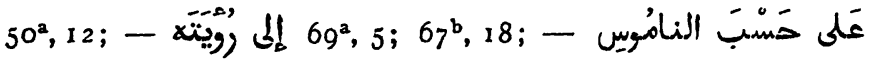

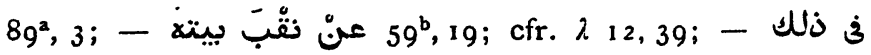

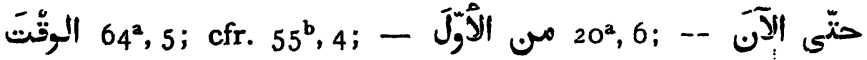
$35^{2}, 16$; u. s. w. 


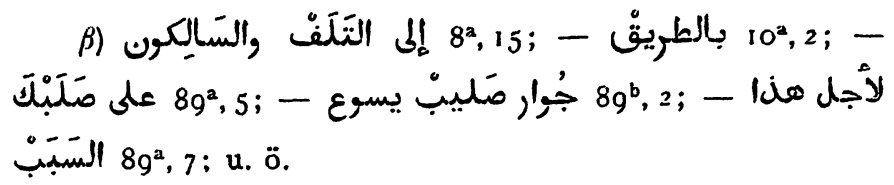

\section{§ 2. Die Adrerbien.}

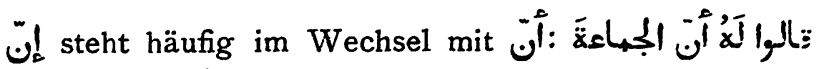
29b , 10 dixerunt ei quia omnes quaerunt te $\mu$ 1, 37;

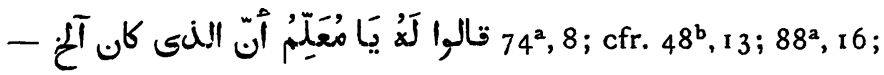
$88^{b}, 17 ; 89^{2}, 18 / 19 ; 89^{b}, 7.9 ;$ u. ö. Dies sind alles Fälle, wo in der Schriftsprache zur Einführung der direkten Rede إن stehen würde, weil die Worte nicht explikativ den Inhalt eines vorhergehenden allgemeinen Ausdrucks wiedergeben und zwar in wörtlicher Rede (cf. WR. II p. 47 B). Vielleicht steht dieser Wechsel im Zusammenhang damit, dass die lateinische oratio recta an den betreffenden Stellen mit quia eingeführt ist.

Sehr häufig werden Sätze und Satzteile durch ثُمَّْ أَنْ

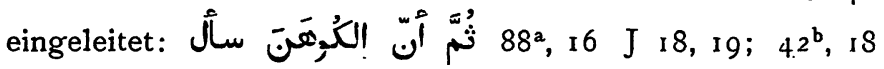
$\mu 15,1$; 16 $6^{b}, 12 \mathrm{M} \mathrm{16,10;} \mathrm{u.} \mathrm{ö.}$

范 $37^{\mathrm{b}}, 13 \mu$ 10, 32. Dasselbe entspricht einem lat. ergo, et - ergo, et, autem, tunc.

إنَّا wird im Sinn unseres deutschen abgeschwächten „nur, denn nur « pleonastisch gebraucht (cfr. Voc., der für إذها nur die Bedeutung imo, BEAU, der für dasselbe auch die von "certes" kennt):

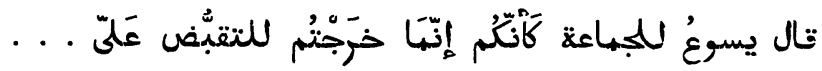
 
Studien über den Codex Arabicus Monacensis Aumer 238. 103 ... comprehendere me $M 26,55$. Ebensowenig hat eine

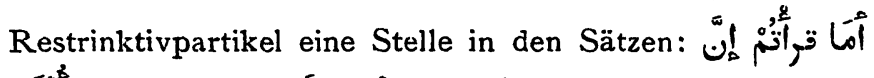

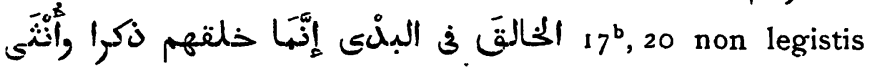
quia qui fecit ab initio, masculum et feminam fecit eos

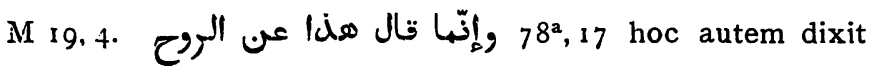
de spiritu $\mathrm{J} 7,39$.

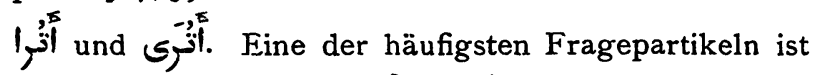
im vorliegenden Texte neben $\hat{l}$ und ${ }^{2}$ die vorausstehende in der doppelten Schreibung; in der Regel entspricht sie

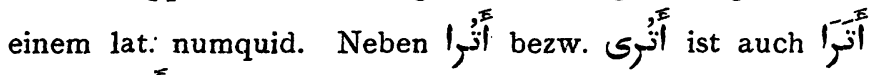

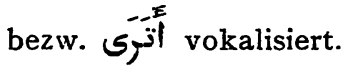

苗 $31^{\mathrm{b}}, 4$ numquid venit lucerna

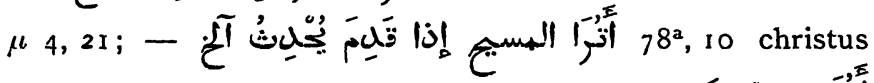

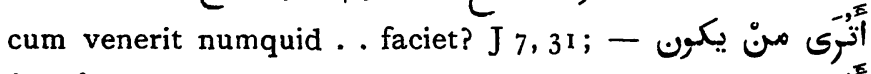

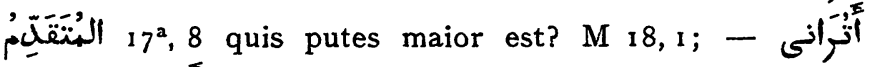
24 20.8 numquid ego sum domine? M 26 ,

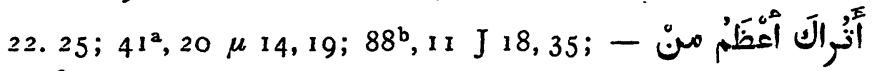
79 أبينا $79^{\text {b }, ~ I ~ n ~ n u m q u i d ~ t u ~ m a i o r ~ e s ~ p a t r e ~ n o s t r o ? ~ J ~ 8, ~ 53 ; ~}$

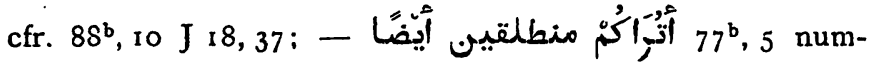
quid et vos vultis abire? J 6,$67 ; 63^{b}, 4 \lambda, 7,24 ; 85^{\mathrm{a}}, 5$ J 16, 19; cfr. $78^{\mathrm{b}}, 3 \mathrm{~J} 7,48 ; 80^{\mathrm{a}}, 12 \mathrm{~J}$ 9, 19; 60 $, 16 \lambda 13,23$;

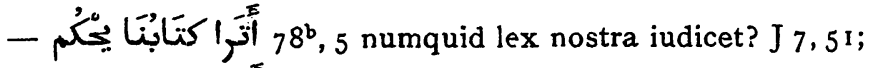
- $79^{2}, 5$ numquid interficiet semet ipsum? J 8, 22. Lat. kennt diese Partikel und gibt: an (numquid)

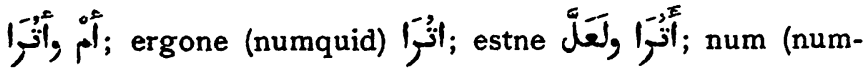


quid) Fabrî 371, 7 und 8 (cfr. Dozy, Suppl. s. أَ)).

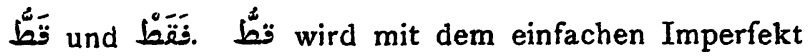

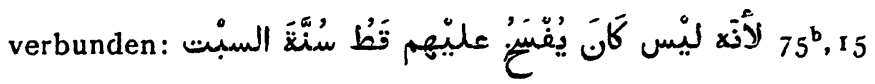
(cfr. quia non solum solvebat sabbatum $\mathrm{J} 5,18$; also eine vom Lateiner abweichende Uebersetzung). Cfr. WK. I p. $286 \mathrm{D}$.

Die Formen von فتطّ sind verschieden; neben فنبس فقطٌ ل non tantum findet sich فيس فتطُ (non tantum)

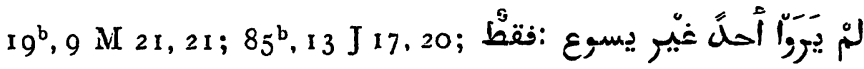

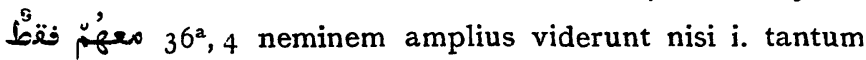

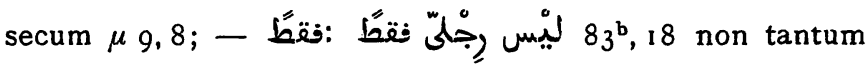
pedes meos $\mathrm{J}$ r 3,9 .

ist meist zur. Bedeutung . von y abgeschwächt und im Wechsel mit ihm gebraucht.

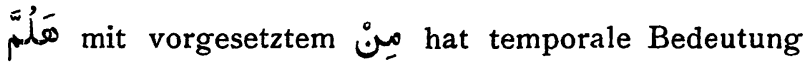
$24^{\mathrm{a}}, \mathrm{I} 2=$ a modo $\mathrm{M} \mathrm{26,29}$, desgleichen mit $22^{\mathrm{a}}, \mathrm{1} 2=$ usque modo $\mathrm{M} 24,2 \mathrm{I}$.

Lo mit dem Demonstrativpronomen verbunden ist Ueber-

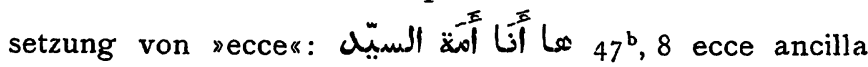

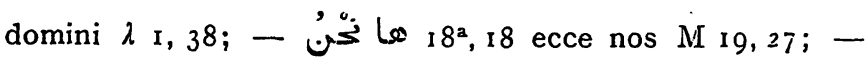

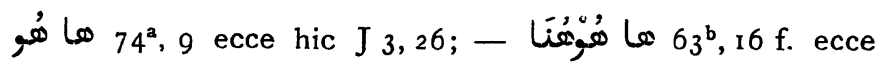
hic (adv.) $\lambda_{17}, 21.23$; - so Voc. sub »ecce

In der gleichen Bedeutung wird Ido verwendet: I

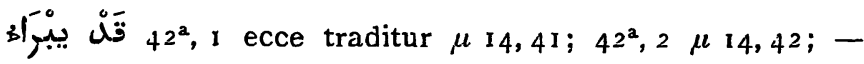


Studien über den Codex Arabicus Monacensis Aumer 238. 105

(omnia)

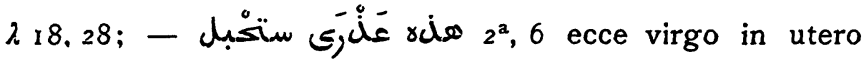
habebit M 2, 23.

Anm. Zum adverbialen Akkusativ ist anzumerken,

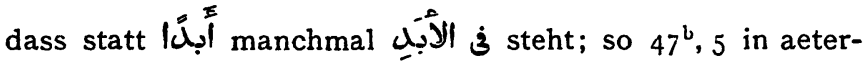

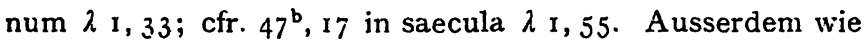
لَ

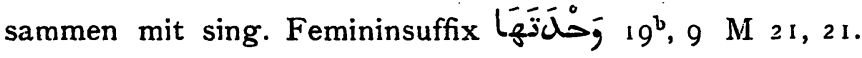

\section{§ 3. Die Konjunktionen.}

إذ in Verbindung mit ذلك steht als Inversionspartikel im Verbalsatz mit der Bedeutung stunc« : فقال إذ ذلَ ذإك وكان إذ ذلك لص $25^{\mathrm{b}}, 4$ habebat autem tunc vinctum etc. M 27, 16 .

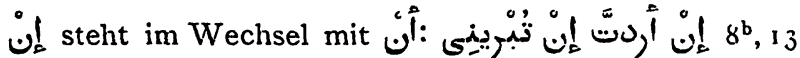

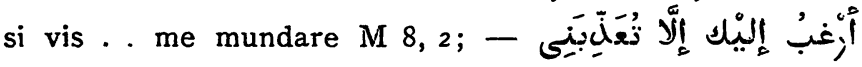
$54^{\mathrm{b}}, 16$ obsecro te ne me torqueas $\lambda 8,28$.

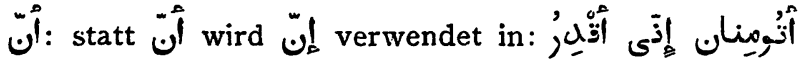

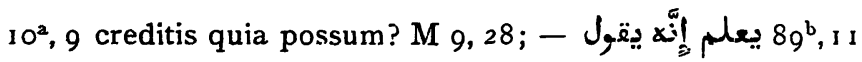

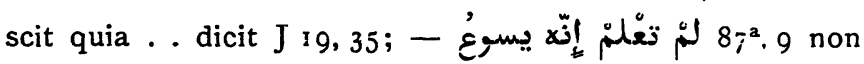
sciebat quia i. est $\mathrm{J} 20,14$.

Pleonastisch erscheint أَّ nach folgenden Konjunk-

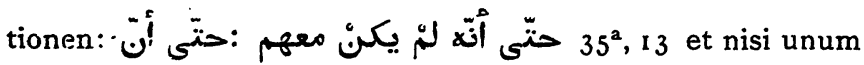

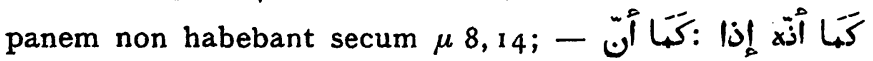

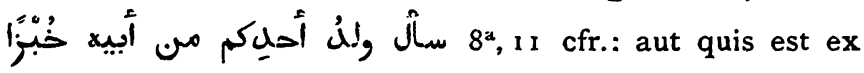


vobis homo quem si petierit filius suus panem numquid

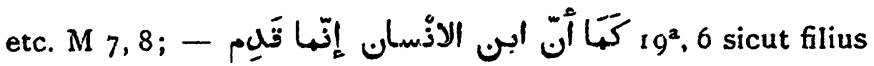
hominis non venit nisi $M$ 20, 28; cfr. $22^{2}, 15 M ~ 24,27$; 85, ro sicut et ego non sum de mundo non rogo $\mathrm{J}_{17}, \mathrm{I}_{4} \mathrm{f}$.

2 $22^{2}, 12$ et nisi breviati fuissent dies illi $M 24,22$.

أن ألّ

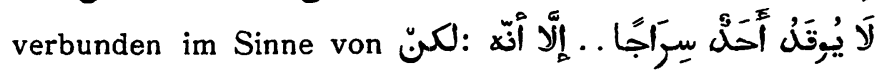

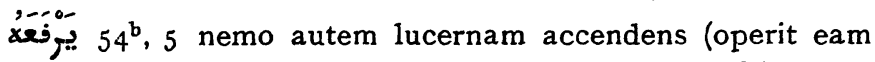
vase) . . sed . . ponit $\lambda 8, \mathrm{r} 6$; cfr. WR. I p. $293 \mathrm{D}^{*}$.

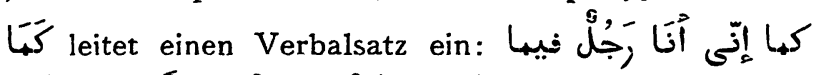

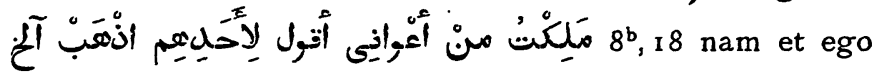
homo sum sub potestate habens sub me milites et dico huic vade etc. $M 8,9$.

An einer Stelle ist $1 \overline{5}$ in temporalem Sinn verwendet: 23º, 15 et effudit super caput ipsius recumbentis $M 26,7$; cfr. WR. II 178 A: "Sometimes

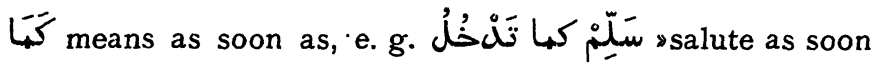
as thou enterest «.

لَّ wird des öfteren von einem pleonastischen gefolgt: I-2 M 4, 2; - - $83^{\text {b, }, ~} 2$ caena facta J I 3,$2 ; 76^{\mathrm{b}}, 10 \mathrm{~J} 6$, I 9 .

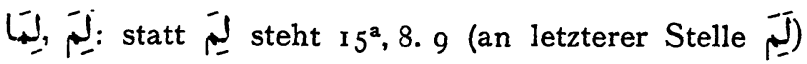
$\bar{\jmath}=$ quare $\mathrm{M}_{\mathrm{I}_{5}, 2 .}$ 
findet sich häufig im Sinne von (temp.), ut, postquam (cfr. unser altertümliches »dieweil « in zeitlichem Sinne): مئو $29^{2}, 14$ postquam autem traditus est i., venit i. $\mu_{1,14}$; $85^{b}$, 20 quae cum dixisset J 18, 1; - 86ª cum intinxisset $\mathrm{J}_{1} 3,26$; u. ö.

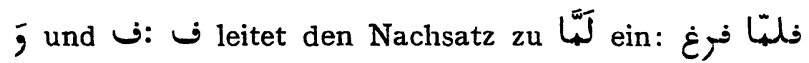
23 il 11 cum consummasset . . dixit M 26, I.

; scheint an einer Stelle = etiam zu stehen:

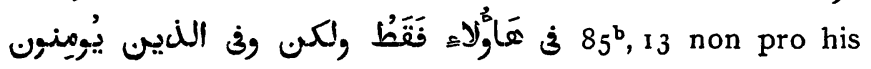
autem rogo tantum sed et pro eis qui credituri sunt $\mathrm{J}_{17}, 20$.

Anm. Zuletzt sei noch auf den eigenartigen pleonastischen Gebrauch von حتبّو الفرضة : حتّى 38 $38^{\mathrm{b}}, 8$ quomodo cum perderent $\mu$ 18,8. Dieser Pleonasmus ist wohl durch eine Ellipse

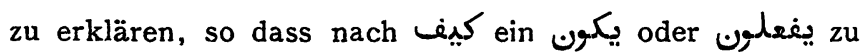
ergänzen wäre.

\section{§ 4. Die Interjektionen.}

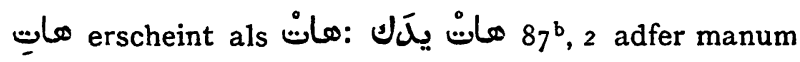
tuam J $20,27$.

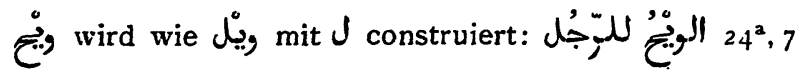
vae homini $M 26,24 ; 21^{a}, 15.17 ; 21^{b}, 1.3 .6$; u. ö. 
يابن

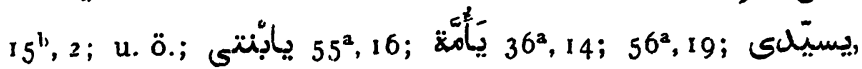

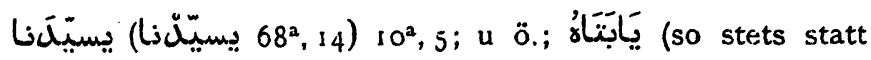
(يابة) $57^{\mathrm{a}}, 14 ;$ u. ö.; u. s. w.

\section{Zur Syntax.}

Die Behandlung der grammatischen Eigentümlichkeiten, soweit sie sich auf die Formenlehre beschränkten, ergab für den behandelten arabischen Text immerhin eine gewisse Sonderstellung, sofern sie ihn als eine Art Mischung aus Schrift-, Umgangs- und mundartlichem Idiom charakterisierten; dabei war jedoch das Ueberwiegen der Schriftsprache augenscheinlich. Dieser Eindruck wird durch die folgende Untersuchung bestätigt werden, wenn gleich auf syntaktischem Gebiet das Verhältnis in der Vermengung wohl dasselbe ist.

\section{Der.Gebrauch der Modi.}

Eine reiche Zahl von Beispielen erweckt den Eindruck, dass im Gebrauch der Modi in seinem Verhältnis zu dem der Schriftsprache eine gewisse Willkür herrscht.

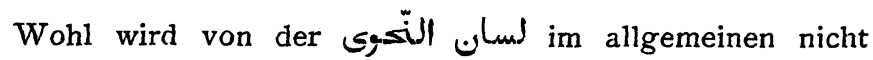
abgewichen, jedoch finden sich Ausnahmen. Einzelne Beispiele, nach Rubriken geordnet, sollen dies im folgenden dartun. 


\section{§ 1. Der Indikativ des Imperfekt.}

a) Der Subjunktiv steht an Stelle des Indikativs.

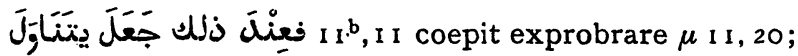
-

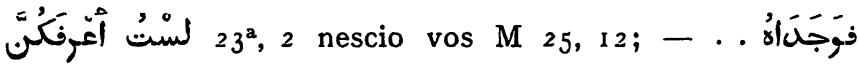
年 $49^{2}, 6$ invenerunt illum in templo sedentem in medio doctorum (audientem

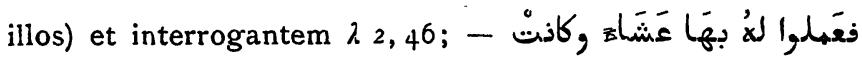

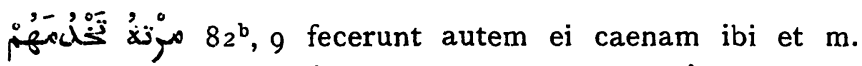

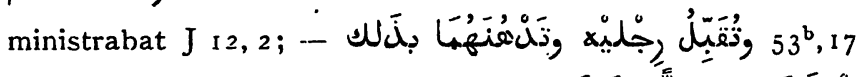

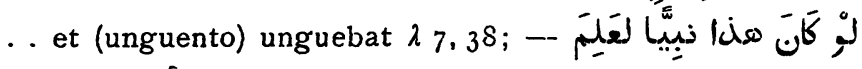

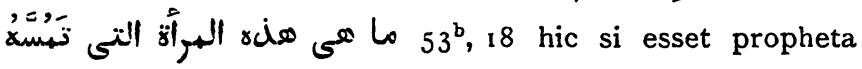
sciret utique quae et qualis mulier quae tangit eum $\lambda$ 7,39;

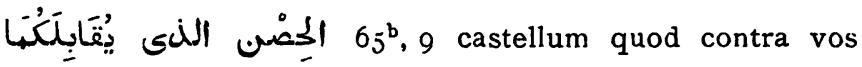
est $\lambda$ 19, 30; - (!) (1) $68^{b} .9$ non novi illum $\lambda 22,58$.

Es sei wiederholt darauf hingewiesen, dass diese Beispiele nur einige wenige aus einer reichen Zahl derselben sind.

b) Der Jussiv an Stelle des Indikativs.

رئِ $14^{2}$, I aperiam in parabolis os meum eructabo abscondita $\mathrm{M} \mathrm{I3}_{13}, 3$; - لئس

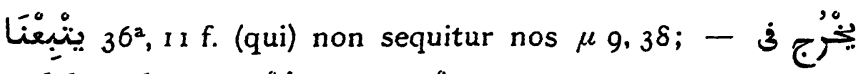
(!) $34^{b}, 4$ in secessum exit purgans omnes esces $\mu 7,19$; cfr. $64^{b}, 1$ र 18,12; -

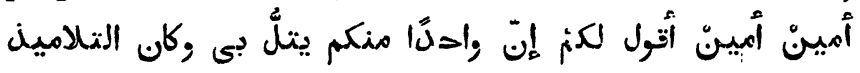


amen amen dico vobis quia unus ex vobis tradet me. aspiciebant ergo ad invi-

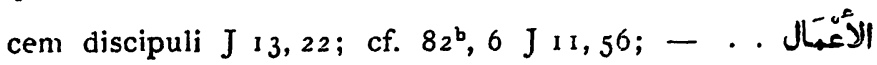
812, 10 opera quae . . facio . haec testimonium perhibent de me sed vos non creditis J 10, 26; cfr. $84^{\text {b }}$, I J 16, 3 .

\section{§ 2. Der Subjunktiv.}

1. Sehr zahlreich sind auch die Fälle, in denen der nach der Uebung der Schriftsprache verlangte Subjunktiv durch einen der andern Modi ersetzt ist.

a) Der Indikativ an Stelle des Subjunktiv

a) nach if etc. mit vorausgehendem Verbum des Befehlens, Wünschens u.s. w.

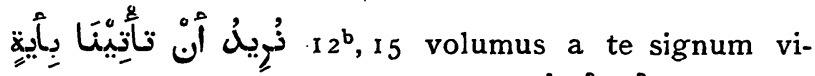

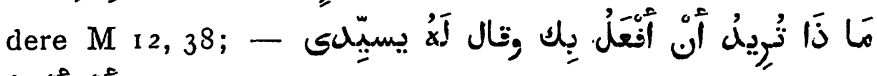

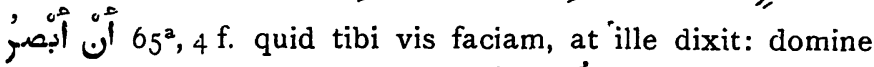

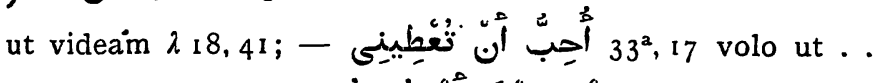

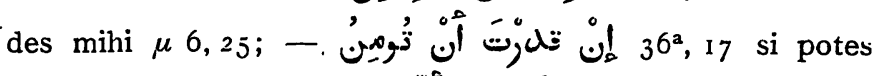

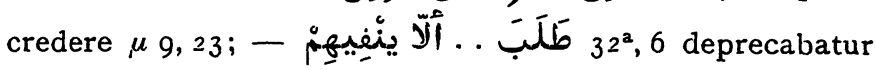
.. ne se expelleret $\mu$, 10; cfr. $54^{b}, 19 \lambda \delta, 31 ; 45^{\mathrm{a}}, 16$;

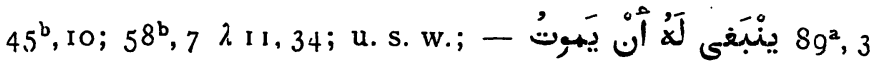

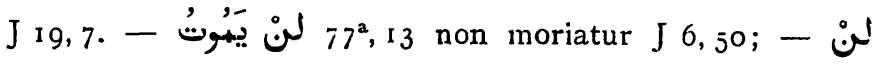
8َ $85^{2}, 5$ non videbitis me J r6, 19;

B) nach $\mathrm{J}, \stackrel{0}{\mathrm{0}}$ etc.

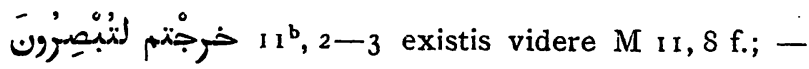


Studien über den Codex Arabicus Monacensis Aumer 238 . 1 I 1 29², 8 cfr. egrediebatur . . et bapti-

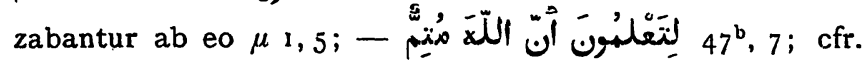

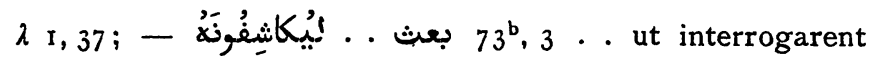
eum J I, I9; cfr. $9^{b}, 11 ; 11^{a}, 17 ; 36^{a}, 16 ; 47^{2}, 14 ; 48^{a}, 10 ;$ $62^{\text {b }}, 8 ; 67^{\mathrm{a}}, 12 ;$ u. s. w.

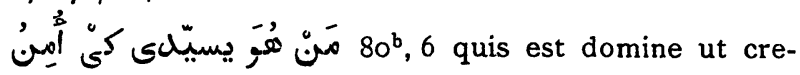

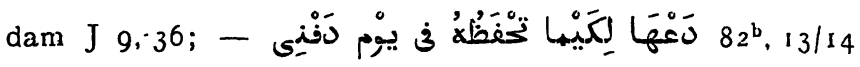
sine illam ut in diem sequlturae meae servet illud $\mathrm{J} 12,7$; - ut et

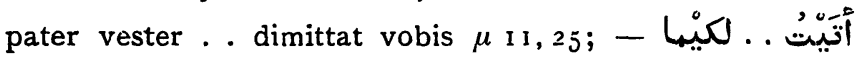
' $72^{\mathrm{b}}, 11$.. cfr. ut manifestetur J 1, 31. In den drei letzten Beispielen ist die Verbindung von finalem $J$ mit dem durch verallgemeinerndes zu beachten.

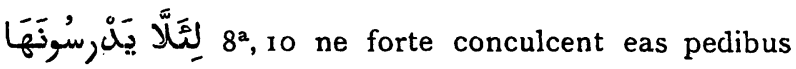
suis $M 7,6 ;$ cfr. $61^{a}, 16 ; 74^{a}, 4 ; 76^{b}, 6$.

Anm. I. كي̣ steht wie in der Schriftsprache auch

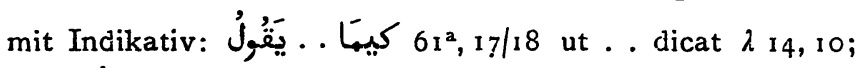
cfr. $40^{\mathrm{b}}$, $19 \mu 13,36$.

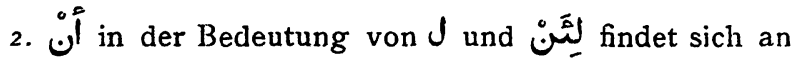
der Stelle 46b, 4 : ut cognoscas - veritatem $\lambda, 1,4$.

3. Das Perfekt steht nach den Finalkonjunktionen, vielleicht um die mit Gewissheit zu erwartenden Geschehnisse antizipierend schon als wirklich darzustellen:

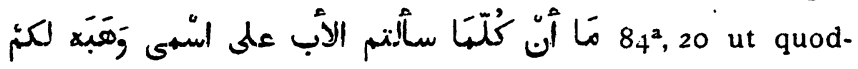


cumque petieritis patrem in nomine meo det vobis $\mathrm{J} \times 5,16$; - $84^{b}$, 5 expedit vobis ut ego vadam $\mathrm{J} 16,7$.

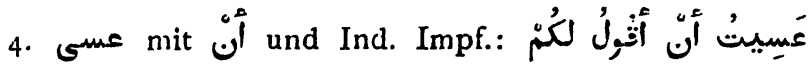
$86^{b}, 1-2$ si quo minus dixissem vobis $\mathrm{J}_{14}, 2$.

b) Der Jussiv an Stelle des Subjunktivs.

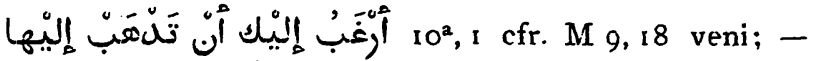
25 , 4 adiuro te . ut dicas nobis M 26,63; cfr. 27 b; 6; 51 $86^{2}$, 19 non potes me sequi $\mathrm{J}$ I 3,36 .

c) Ein dritter Fall ist derjenige, dass bei zwei oder mehreren abhängigen Verben das erste im Subjunktiv steht, die anderen aber in einem anderen Modus folgen: 5a , I ut videant vestra bona opera et glorificant patrem vestrum $M$ 5, 16;

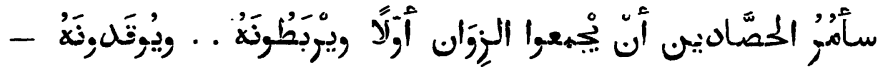
I $3^{b}$, I $5 \mathrm{f}$. dicam messoribus: colligite primum zizania et

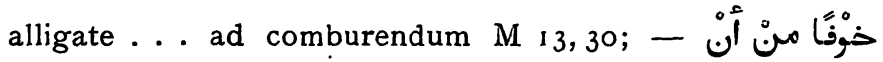

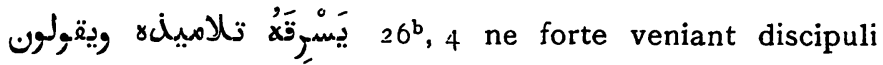
eius et furentur eum et dicant $\mathrm{M} \mathrm{27}_{27}, 64$ cfr. $19^{2}, 6 \mathrm{M} 20$, $28 ; 62^{\mathrm{b}}, 4 \lambda_{15}, 24 ; 84^{\mathrm{a}}, 15 \mathrm{~J} \mathrm{I} 5,11 ; 89^{\mathrm{b}}, 87 \mathrm{~J} 19.31 .-$

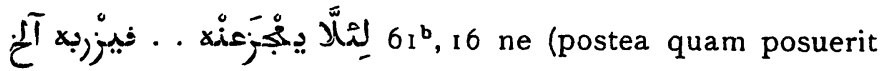
fundamentum et) non potuerit perficere . . incipiant in-

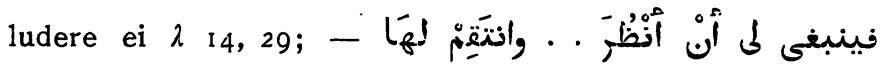
$64^{\mathrm{a}}, 14$; cfr. $\lambda 18,5$ et vindicabo illam. 
2. a) أن wird vor dem Subjunktiv ausgelassen. Dasselbe geschieht verhältnismässig häufig; das abhängige Verbum folgt dann sowohl im Indikativ als auch im Subjunktiv; am häufigsten tritt dieser Fall ein bei إن $24^{\mathrm{b}}, 5$ si non potest hic

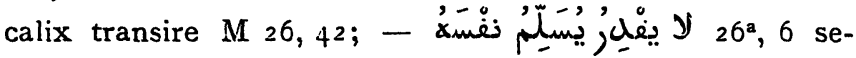
ipsum non potest salvum facere $\mathrm{M} \mathrm{27,42;-}$

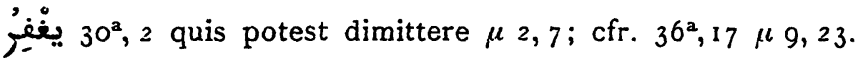

Etwas anders ist die Sachlage, wenn das abhängige Verbum von einem Zeitwort des Befehlens, Wünschens etc. regiert ist. In der Schriftsprache kommt dieser Fall selten vor. Wenn er eintritt, so steht das abhängige Verbum im Indikativ oder Subjunktiv; ersteren verlangt Beiḑàwî (zu Ķor'ân II 77; cfr. Ḥamâsa p. 438 Z. 6 sq., LaNE p. 104 c; zum Ganzen vergl. WR. II p. 27 A und $D^{*}$ ). In der Hs. finden sich folgende Beispiele mit Indikativ:

50ª 7 dic ut hii lapides

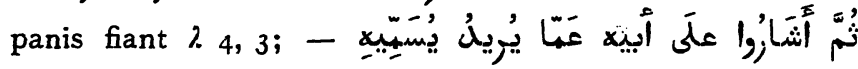
$48^{\mathrm{a}}$, I innuebant autem patri eius quem vellet vocari illum 2. 16, 2; vultis audire? $\mathrm{J} 9,27$.

[b) Hier soll noch ein Beispiel angeführt werden, wo das regierende Zeitwort ein Verbum der Annäherung

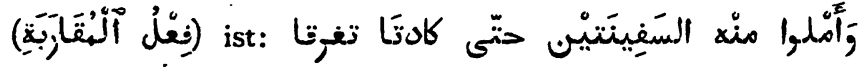
$51^{2}, 12\left(M^{2}\right)$ et impleverunt ambas naviculas ita ut (pene) mergerentur $2.5,7$. 


\section{§ 3. Der Jussiv.}

Auch in der Verwendung des Jussivs lässt sich ein Schwanken in der Weise wahrnehmen, dass er, wo ihn die Schriftsprache fordert, teilweise durch den Indikativ oder Subjunktiv ersetzt ist.

a) Statt eines Jussivs mit $\boldsymbol{y}$ zur Bezeichnung des verneinenden Imperativs stehen sowohl Indikativ als Subjunktiv: تَكُونُ

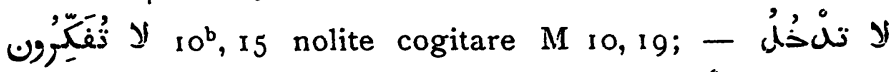

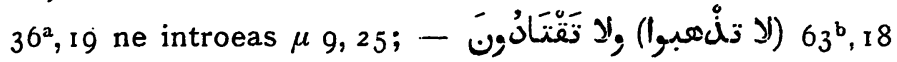
(nolite ire) nec sectemini $\lambda_{17}, 23$; cfr. $64^{\mathrm{a}}, 5 \lambda_{17}, 31 ; 73^{\mathrm{b}}$,

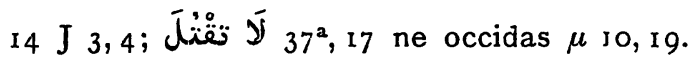

b) Weit zahlreicher sind die Abweichungen bei Hierbei ist ein Dreifaches zu beobachten: einmal dass zur Verneinung eines Geschehens oder einer Handlung in der Vergangenheit neben dem Jussiv der Indikativ, sodann auch der Subjunktiv verwendet wird, endlich dass in einem Falle die Verwendung von is mit Indikativ zur Verneinung der Zukunft angenommen werden muss.

a)

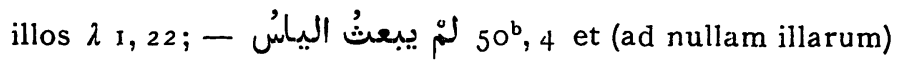
missus est helias $\lambda 4,20 ;-$ - et

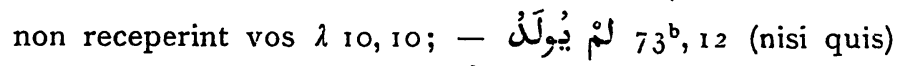

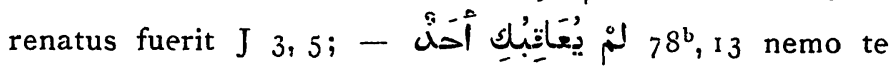

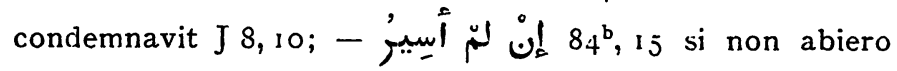
J 16, 7; cfr. $33^{\mathrm{a}}, 3-4 \mu 6$, I1; $36^{\mathrm{b}}, 2, \mu 9,28 ; 50^{\mathrm{b}}, 3-4$

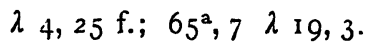


B) $10^{b}, 10$ et quicumque non receperit

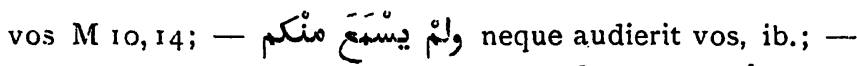

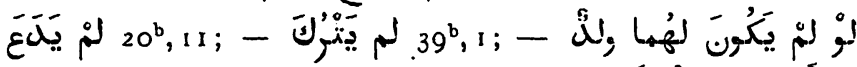
$47^{2}, 4$ et non erat illis filius $\lambda_{1}, 7 ;-\hat{j}^{2}$ $53^{2}, 8$ me ipsum non sum dignus arbitratus $i, 7,7 ;-$ il

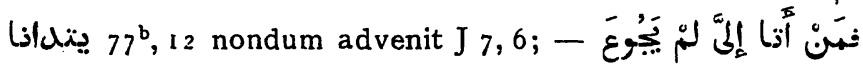

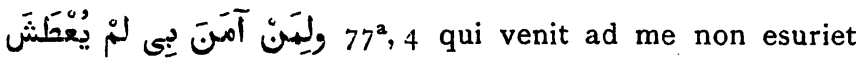
et qui credit in me non sitiet $\mathrm{J} 6,35$; cfr. $36^{\mathrm{b}}, \mathrm{I} 3 \mu 9,40$; $38^{\mathrm{a}}, 13 \mu \mathrm{II}, 2 ; 55^{\mathrm{a}}, 18 \lambda 8,5 \mathrm{I} ; 55^{\mathrm{b}}, 6 \lambda 9,5 ; 68^{\mathrm{a}}, 10 \lambda 22,34$; $72^{\mathrm{a}}, 15 \mathrm{~J} \mathrm{I}, 13 ; 72^{\mathrm{b}}, \lambda .5,5$.

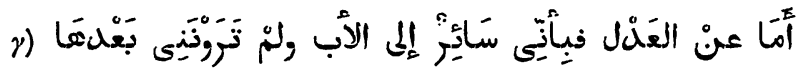
$84^{\mathrm{b}}, \mathrm{I} 7$ de iustitia vero: quia ad patrem vado et iam non videbitis me $\mathrm{J} 16,10$ (wenn nicht mit einigen Hss. videtis gelesen wird).

\section{§ 4. Der Imperativ.}

Es ist schon in meiner Diss. (S. 22 f.) die Vermutung ausgesprochen worden, dass in einigen vokalisch auf $a$ auslautenden Formen des Imperativs 2. p. s. m. vielleicht verkürzte energische Bildungen vorliegen; cfr. $3^{b}$, Io vade

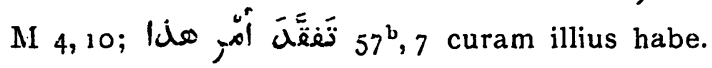

Wenn gelegentlich die Form des Indikativs steht تَ $63^{2}, 14$ recordare $i 16,24$-, so darf wohl daraus nicht zuviel geschlossen werden, etwa in der Richtung, dass, wie im heutigen Marokkanischen zur Bezeichnung des Imperativs der 3. und 1. pass., das einfache Imperfekt verwendet wurde (cfr. J. Rud. p. 287 no. 185); eher dürfte darin eine abgeschwächte Form des Imperativs gesehen werden. 
2. Die Rektion des Nomens.

\section{a) Der Akkusativ.}

a) Der Akkusativ als Objekt.

Bei der Besprechung der Kasusflexion des Nomens habe ich schon Diss. (S. 5o ff.) auf die willkürliche Verwendung der Flexionsendungen aufmerksam gemacht. Hier sei diese Erscheinung von syntaktischen Gesichtspunkten aus verwertet. Speziell für dieses Kapitel heisst dies, dass die Flexionsendungen des Nominativs oder Akkusativs bei Nomina. sich finden, die in ihrem syntaktischen Verhältnis ein erstes oder zweites Objekt (الهفعول الثانى ,الهغعول الأُول) darstellen. Zugleich sei hier auf die Diss. 1. c. versuchte Erklärung dieser Erscheinung hingewiesen. Einzelne wenige Beispiele derselben sind:

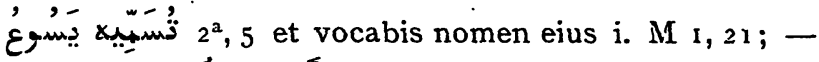

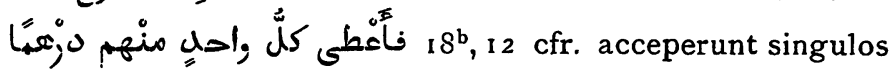

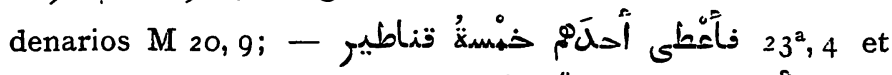

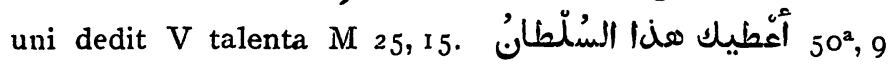
tibi dabo potestatem hanc $\lambda 4,6$; cfr. $45^{\mathrm{b}}, \mathrm{r} \lambda$ cap. X;

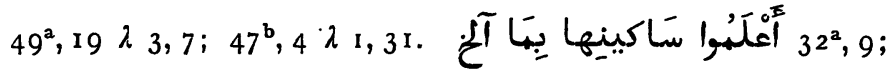
cfr. $\mu 5, \mathrm{I} 4$.

Aehnlich liegt der Fall, wenn das Verbum passiv kon-

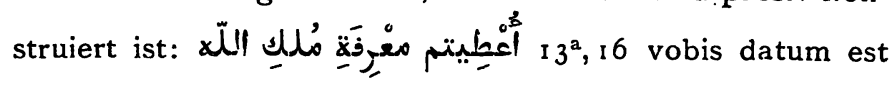
nosse mysteria regni caelorum; ebenso $3 \mathrm{I}^{2}, \mathrm{I} 6 \mu 4, \mathrm{Ir}$.

Cfr. hier auch den doppelten Nominativ beim Passi-

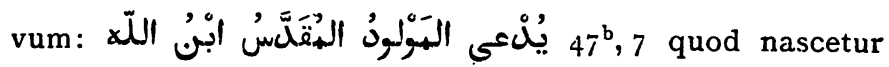
sanctum uocabitur filius dei $\lambda, 35$. 
B) Der Akkusativ als allgemeines Objekt

$$
\text { (الهفْعرل الإُطْلَققى). }
$$

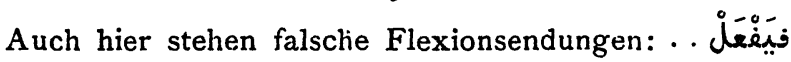

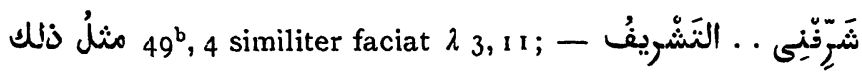
الذ $85^{b}, 2$ clarifica me . claritate J 17,5 .

Besonders häufig steht der Nominativ bei den zur Vergleichung verwendeten komparativen Af al-Formen:

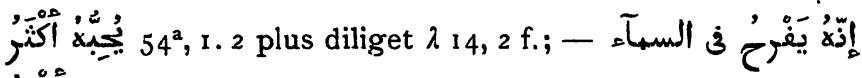
أَكْثَ $62^{2}, 7 / 8$ ita gaudium erit in caelo (plus) quam 2. 15,7 ; u. ö.

r) Die Rektion des nomen verbi, agentis et patientis.

Eigentümlich ist die Verbindung des Subjekts- und Objekts-Nomens mit dem nom. verb. ohne jede Präposition bei einem der beiden:

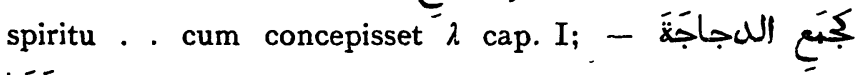
فرارِجَبَا $2 \mathrm{I}^{\mathrm{b}}, 16 \mathrm{f}$. quemadmodum gallina congregat pullos suos $M$ 23, 37 .

Das Objekts-Komplement steht im Nominativ : ل $46^{2}, 11$; cfr. $\lambda$ cap. XVIII ac traditorem desig-

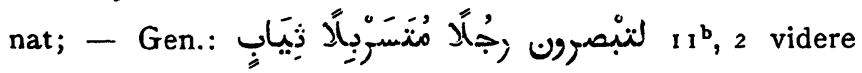
hominem (mollibus) vestitum $\mathrm{M}_{\mathrm{I}}, 8$.

d) Der Akkusativ nach أنّ und Schwestern.

Die Fälle sind zahlreich, in denen nach أَّ und seinen Schwestern das auf sie folgende Nomen im Nominativ steht: 


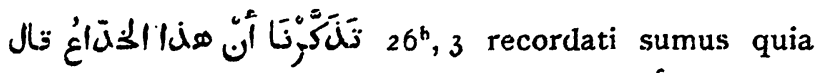
seductor ille dixit M 27,62; - 3332,6

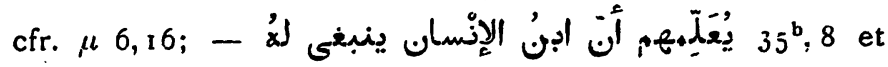
coepit docere illos quoniam oportet filium hominis (multa

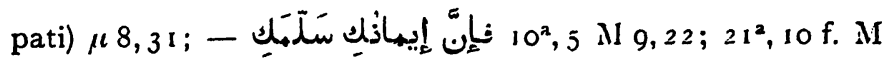

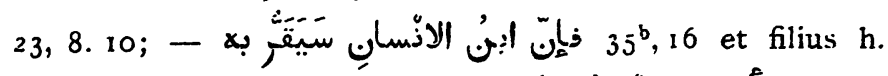
"confitebiturs cum $\mu$ 8, 38 ; $\lambda \times 6,28$.

Andererseits stets das Subjektsnomen nach بيني im

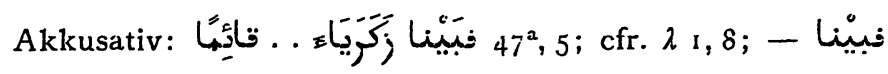

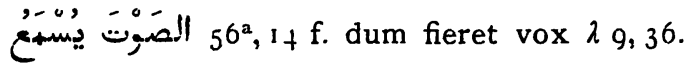

e) Der Akkusativ beim Vokativ.

Der Akkusativ steht nach $ي$, wo die Schriftsprache

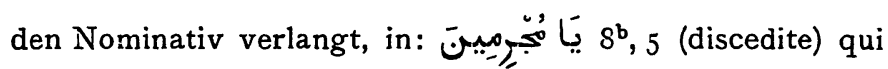
operamini iniquitatem M 7,23; - $55^{2}, 20$ puella surge $\lambda 8,54$.

Umgekehrt findet sich der Nominativ (bezw. Genetiv), wo in jener der Akkusativ zu stehen kommt: يَا نَنْنُ 1ألفاعى 12b 1 I progenies viperarum M 12,34; cfr. $12^{\mathrm{b}}, 15$

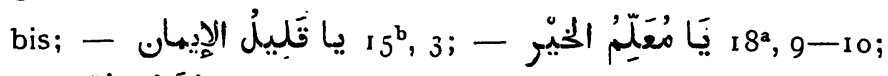

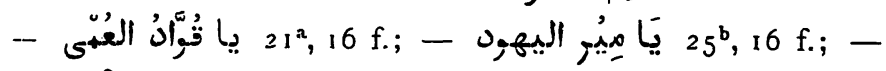

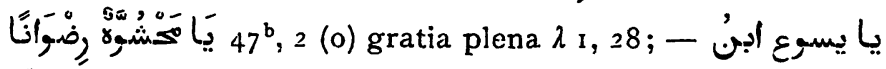

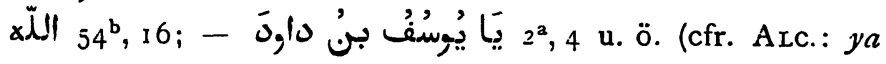

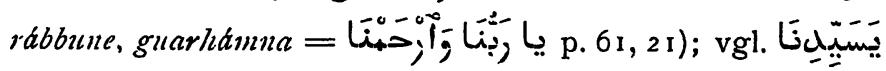
$64^{2}, 9$ domine $\lambda_{17}, 36$. 
5) Der accusativus adverbialis.

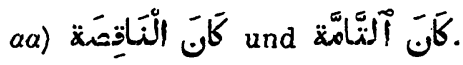

Offenbar wurde nicht mehr scharf unterschieden $z$ wischen den beiden Bedeutungen von $\overline{-} \bar{\zeta} ;$ denn einerseits hat das كان التَّامََّّ des öfteren sein logisches Subjekt im Akkusativ nach sich wie in den Beispielen: فلنّا كاب ذات يُون $33^{\mathrm{a}}, 12$ et cum dies opportunus accidisset $\mu 6,12$; -

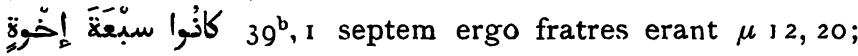

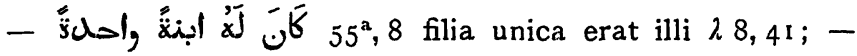
andererseits nimmt das كان الناقصة sehr häufig das Prädikat im Nominativ zu sich; die Fälle sind sehr zahlreich, es folgen einige Beispiele:

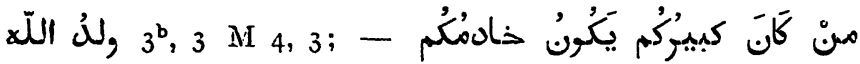
$21^{2}, 1$ I qui maior est vestrum erit minister vester $M 2_{3}, 11$; كانت - 26, 24; 29 socrus

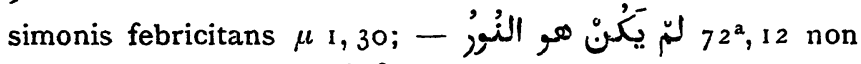

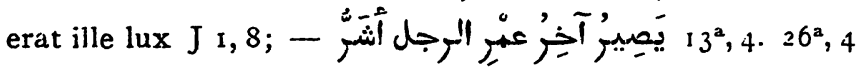

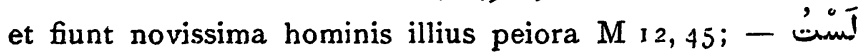

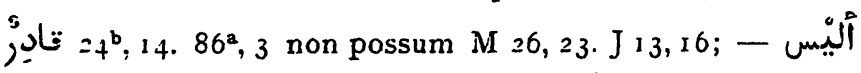
32b, 10 $\mu$ 5. 39.

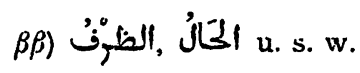

Statt des accus. adverb. stehen andere Kasus, häutig zur Bezeichnung: 


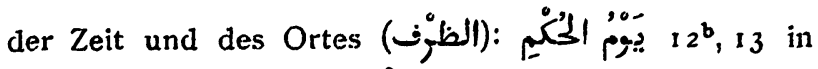
die iudicii M 12,36; - أيّام 12 $12^{\mathrm{b}}, 16$ tribis diebus

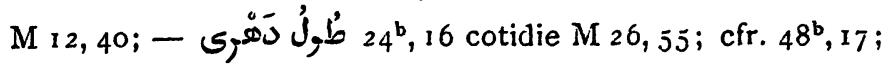

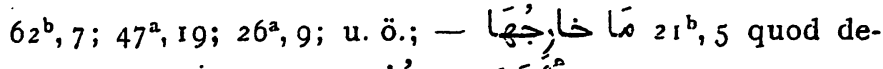

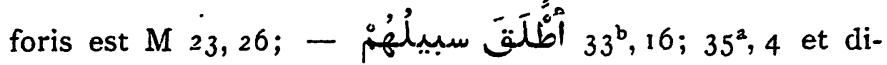
misit eos $\mu$ 8,9; -

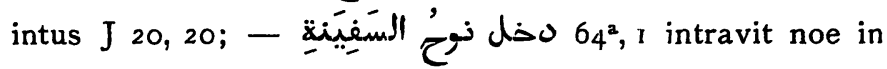
arcam $\lambda_{17}, 29 ;$ u. s. w.;

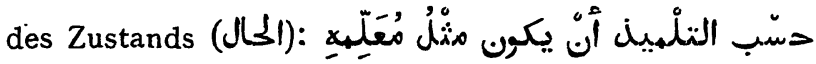
$10^{b}, 20$ sufficit discipulo ut sit sicut magister eius $\mathrm{MI}$ 10, 25;

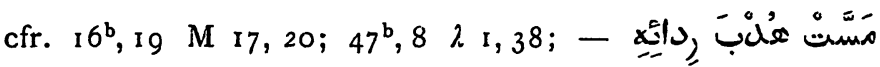
تَأِيلٌٌ $10^{2}, 3$ tetigit fimbriam vestimenti eius; dicebat enim M 9, 21; - 29ª 10 vidit apertos cae-

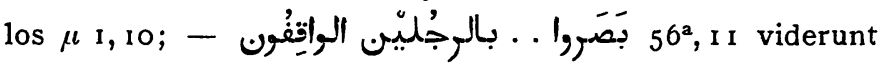
duos viros qui stabant $\lambda 9,32$; cfr. $55^{\mathrm{a}}, 3 \lambda 8,35 ; 57^{\mathrm{b}}, 5$

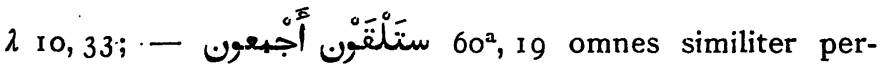

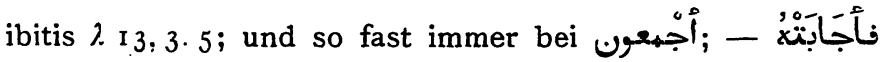
25 البكماءةُ كُلِّها $25^{b}, 12$ et respondens universus populus (dixit) M 27,25; - $34^{\text {a }}$, 9 audite omnes $\mu$ 7, I4;

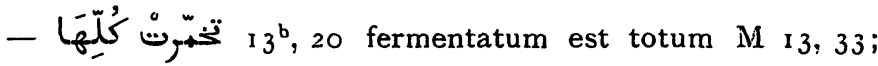
.cfr. hierzu Alc. s. stoda cosa cullixéi (= كُلّلَيْيَ

Anm. Eine Art instrumentaler Akkusativ scheint

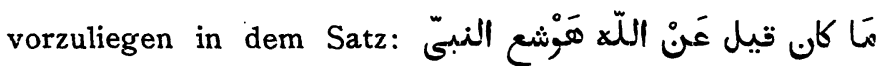
$2^{\mathrm{b}}, 4$. 5 quod dictum est a domino per (H.) prophetam $\mathrm{M} \mathrm{2,} 5$. 
3. Die Verbindung des Nomens.

Es ist noch erforderlich, auf die Frage der Verbindung der Nomina unter einander mit einigen Worten einzugehen, da sich im Texte einzelne der Schriftsprache gegenüber sich als Abweichungen charakterisierende Eigentümlichkeiten finden. Sie bilden jedoch in ihrer Stellung zur Gesamtheit der betreffenden syntaktischen Fragen nur Ausnahmen.

\section{§1. Permutativ (بََ).}

Hierbei kommt die Konstruktion von ${ }^{4}$ etc. in Be-

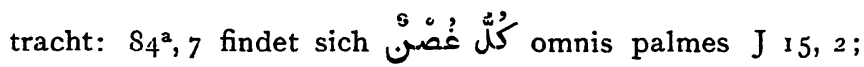
- $34^{b}, 3$ in secessum exit purgans omnes escas $\mu$ 7, 19. Bei 5 ist

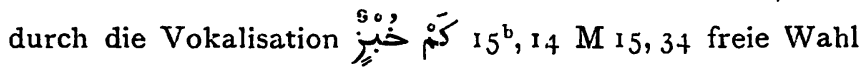
gelassen.

\section{§ 2. Qualifikation.}

Besondere Beachtung verdient, dass in zahlreichen Fällen das pronomen demonstrativum und sein Nomen im

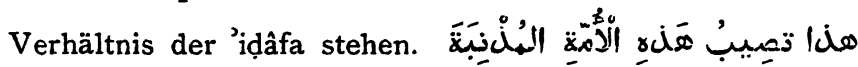

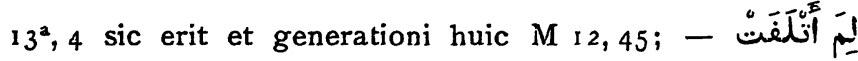

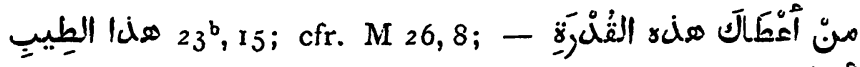
$38^{\mathrm{b}}, 19$ quis tibi dedit hanc potestatem? $\mu$ 11, 28; - أحلَ الخ

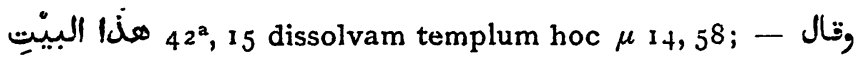

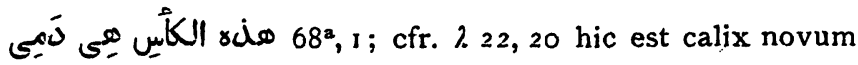

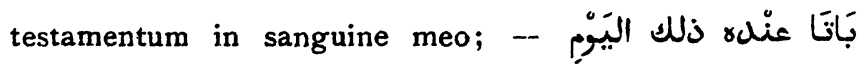


$72^{b}, 16$ apud eum manserunt die illo J 1, 39; cfr. $24^{a}, 15$; - $83^{\mathrm{a}}, 3$ (audierunt) eum fecisse hoc signum J 12,18; cfr. $14^{\mathrm{a}}: 9 \mathrm{M} \mathrm{I}_{3}, 44 ; 66^{\mathrm{a}}, 5 \lambda 20,2 ; 82^{\mathrm{b}}, 1 \mathrm{I}$

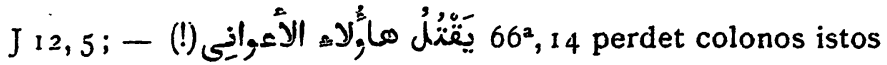
$\lambda 20$, ra.

Tritt ein Adjektiv, nom. ag. oder pat, als Qualifikation zum Stamm, so richtet sich dasselbe nicht immer nach dem letzteren.

50ª , 8 (praedicare) annum domini acceptum $\lambda_{4}, 19 ;-$ - $56^{2}, 18$ spiritus immundus adprehendit eum $\lambda$, أبُْ يُبرسفَ

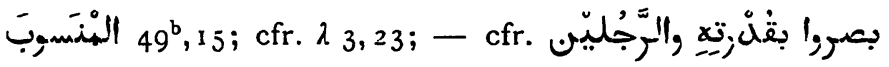
56a , I . . . et duos viros qui stabant cum illo $\lambda$ 9,32; - ' - $47^{2}, 3$ fuit in die-

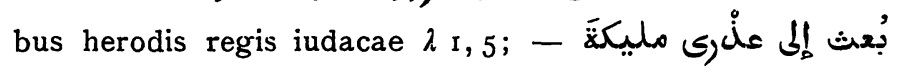
(ج) $47^{\mathrm{b}}$, I missus est ad virginem desponsatam viro $\lambda$ I, 27; cfr. $16^{\mathrm{a}}, 9$ M.16, $14 ; 53^{\mathrm{b}}, 8 \lambda 7,28$.

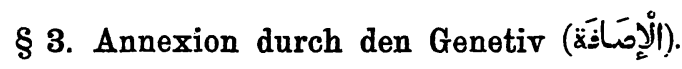

Ueber die Willkür in der Vokalisation bei dem bestimmenden Nomen ist Diss. (S. 52) gehandelt worden. An dieser Stelle sollen einige Beispiele genannt werden, wo das durch den Genetiv bestimmte Nomen den Artikel behält, ohne dass das bestimmende Nomen als النَّابِ aufgefasst werden könnte. 
Studien über den Codex Arabicus Monacensis Aumer $238 . \quad 123$

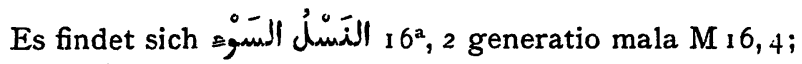
-

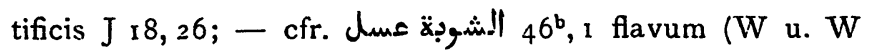
306 Z. I2); event. ist aber hier عسل späterer erklärender Zusatz des wenig bekannten الشوبر

غيْر schliesst an einigen Stellen sein Nomen im gleichen Kasus an sich: لَال يعلم أحنَ منْ هور الأبب غيْر الإببن

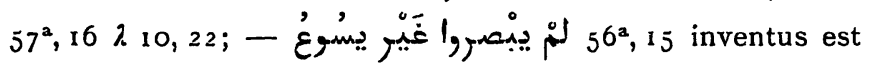
iesus solus $\lambda, 36$.

Anm. Vgl. noch die seltenen Fälle, wo ein Präpositionalausdruck so mit dem Nomen verbunden ist, dass

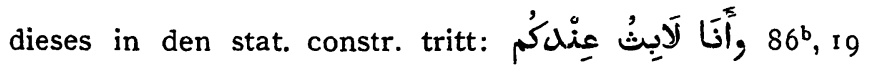
(locutus sum) manens apud vos J 14, 25; cfr. يَثْوضُون فِبِ

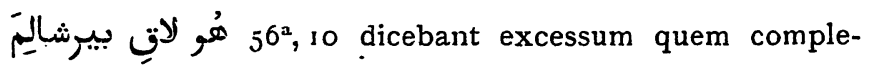
turus esset in i. $\lambda .9,31$.

\section{Zur Satzlehre.}

Die Erörterung über dieses Kapitel kann sich auf ein paar kurze Bemerkungen über einige abweichende Satzkonstruktionen beschränken.

\section{§ 1. Verbalsatz.}

Gegen die Schriftsprache verstösst der Gebrauch der männlichen Form des Verbums in den Sätzen: جَعَل يَتَنَارَل

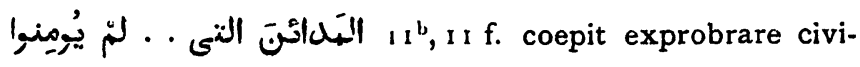


tates... (quia non egissent paenitentiam) M II, 20; 年 tur bethleem $\lambda 2,4 ;-$ - $64^{2}, 8 / 9$ et altera mulier relinquetur 217,35 .

Der Dual ist zu erwarten in folgenden Sätzen: Léco, (duo caecos) et ait quid vultis ut faciam vobis? $M 20,32$; -

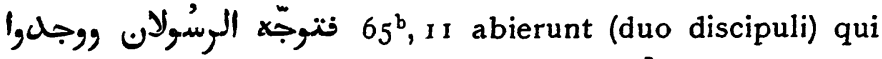

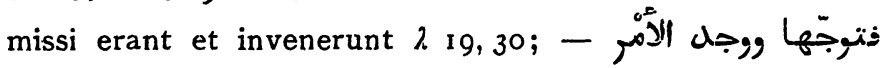

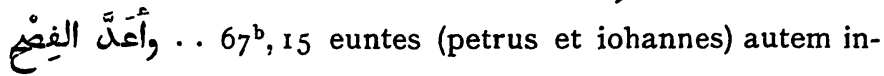
venerunt sicut dixit illis et paraverunt pascha $\lambda 22,12$ (vielleicht ist im letzten Satze beidemal das Verbum mit dem folgenden mit I beginnenden Wort zusammenge-

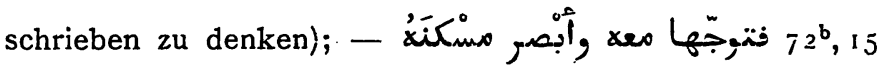
venerunt (duo discipuli) et viderunt ubi maneret J I, 39.

\section{§ 2. Der Nominalsatz.}

Vereinzelt liegt der Fall, dass das Prädikatsnomen (الهبتنبأ) mit seinem Subjekt (الكبر) Kasus nicht übereinstimmt: قُولوا خُهُ sumus $\lambda$ I7, 10. Ebenso vereinzelt bezüglich der Ueber-

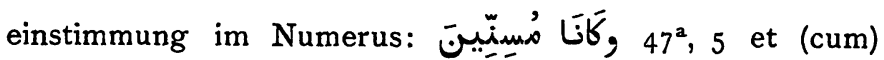
ambo processissent (in diebus suis) $\lambda_{1,7}$.

Auf falscher Analogie beruht es, wenn das Prädikats-

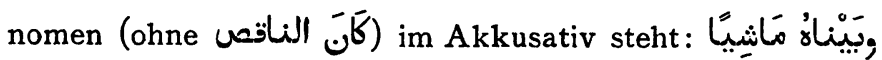

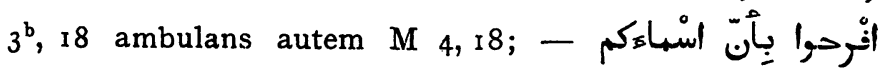


Studien über den Codex Arabicus Monacensis Aumer 238. $\quad$ I 25

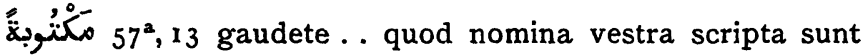
2. 10, 20; - - $66^{2}, 6$ si dixerimus de caelo (sc. esse baptismum) $\lambda 20,5$.

\section{§ 3. Verschiedene andere Satzarten.}

Die einen exzeptiven Satz einführenden Worte haben verschiedene Konstruktionen.

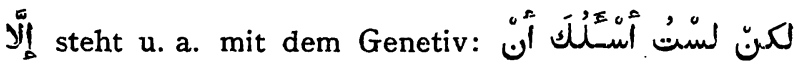
68 68 a 17 . . non mea voluntas sed tua fiat $\lambda 22,42$.

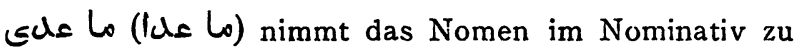

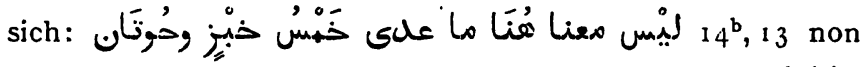
habemus hic nisi quinque panes et duos pisces; - ستُغْغَرْ

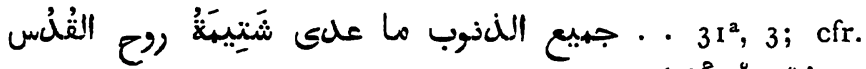

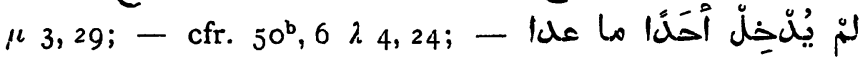
$55^{\mathrm{a}}, 18$ non permisit intrare secum quemquam nisi petrum $\lambda 8,5 \mathrm{I}$.

(Fortsetzung folgt.) 